Linköping Studies in Science and Technology

Licentiate Thesis No. 1868

\title{
On Massive MIMO for Massive Machine-Type Communications
}

\author{
Ema Becirovic
}


Linköping Studies in Science and Technology

Licentiate Thesis, No. 1868

\section{On Massive MIMO for Massive Machine-Type Communications}

Ema Becirovic

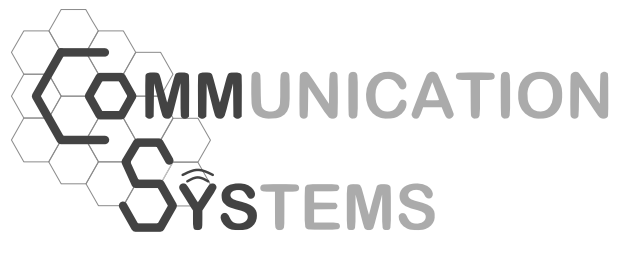

Division of Communication Systems Department of Electrical Engineering (ISY) Linköping University, 58183 Linköping, Sweden www.commsys.isy.liu.se

Linköping 2020 
This is a Swedish Licentiate Thesis.

The Licentiate degree comprises 120 ECTS credits of postgraduate studies.

On Massive MIMO for Massive Machine-Type Communications

(c) 2020 Ema Becirovic, unless otherwise stated.

ISBN 978-91-7929-915-6

ISSN 0280-7971

URL http://urn.kb.se/resolve?urn=urn:nbn:se:liu:diva-162586

Printed in Sweden by LiU-Tryck, Linköping 2020 


\section{Abstract}

To cover all the needs and requirements of mobile networks in the future, the predicted usage of the mobile networks has been split into three use-cases: enhanced mobile broadband, ultra-reliable low-latency communication, and massive machine-type communication. In this thesis we focus on the massive machine-type communication use-case which is intended to facilitate the ever increasing number of smart devices and sensors.

In the massive machine-type communication use-case, the main challenges are to accommodate a huge number of devices while keeping the battery lives of the devices long, and allowing them to be placed in far-away locations. However, these devices are not concerned about other features such as latency, high data rate, or mobility.

In this thesis we study the application of massive MIMO (multiple-input multiple-output) technology for the massive machine-type communication use-case. Massive MIMO has been on the radar as an enabler for future communication networks in the last decade and is now firmly rooted in both academia and industry. The main idea of massive MIMO is to utilize a base station with a massive number of antennas which gives the ability to spatially direct signals and serve multiple devices in the same time- and frequency resource.

More specifically, in this thesis we study A) a scenario where the base station takes advantage of a device's low mobility to improve its channel

estimate, B) a random access scheme for massive machine-type communication which can accommodate a huge number of devices, and $\mathbf{C}$ ) a case study where the benefits of massive MIMO for long range devices are quantified. The results are that the base station can significantly improve the channel estimates for a low mobility user such that it can tolerate lower SNR while still achieving the same rate. Additionally, the properties of massive MIMO greatly helps to detect users in random access scenarios and increase link-budgets compared to single-antenna base stations. 


\section{Populärvetenskaplig Sammanfattning}

Den förväntade användningen av framtidens mobila nätverk har delats upp i tre användningsområden för att kunna täcka alla förutspådda behov. I den här avhandlingen fokuserar vi på användningsområdet mMTC (massiv maskintypskommunikation, eng: massive machine-type communication) som är ämnat för att hantera det ökande antalet av smarta enheter och sensorer.

De största utmaningarna i mMTC-fallet är att tillgodose ett enormt antal enheter samtidigt som batterierna på enheterna ska räcka länge och enheterna ska tillåtas att placeras på avlägsna platser. Däremot är andra egenskaper som latens, datatakt och mobilitet relativt oviktiga för dessa enheter.

I den här avhandlingen kollar vi på hur vi kan använda massiv MIMO (eng: multiple-input multiple-output) för mMTC-fallet. Massiv MIMO har det senaste årtiondet varit på tapeten som en av de största möjliggörarna för framtida kommunikationsnätverk och har nu en stark grund i både akademin och industrin. Massiv MIMO utnyttjar ett stort antal antenner vid basstationen för att kunna rikta signaler och tjäna många enheter vid samma tid och frekvens.

Mer specifikt, studerar vi $\mathbf{A}$ ) ett fall där basstationen utnyttjar en enhets låga mobilitet för att förbättra kanalskattningarna, B) ett förslag för att hantera tillträde av enheter till nätverket som kan tillgodose ett stort antal enheter samt $\mathbf{C}$ ) en fallstudie där vinsterna av massiv MIMO för enheter på långa avstånd är kvantifierade. 


\section{Contents}

Acknowledgements $\quad$ vii

List of Abbreviations $\quad$ ix

1 Introduction and Motivation $\quad 1$

1.1 Contributions of the Thesis . . . . . . . . . . . . 3

1.2 Excluded Papers . . . . . . . . . . . . 5

2 Massive MIMO $\quad 7$

2.1 System Model . . . . . . . . . . . . . . . . . . 7

2.1 .1 Reciprocity . . . . . . . . . . . . . 8

2.1.2 Coherence Block . . . . . . . . . . . . . . . . 9

2.1.3 Channel Model . . . . . . . . . . . . . . . . . . . 9 9

2.2 Channel Estimation . . . . . . . . . . . . . . . . 11

2.3 Spatial Multiplexing . . . . . . . . . . . . . . . . 13

2.4 Capacity Bounds . . . . . . . . . . . . . . . . . . . . 14

3 Massive Machine-Type Communications $\quad 17$

3.1 Assumptions . . . . . . . . . . . . . . . . 17

3.2 Massive MIMO for mMTC . . . . . . . . . . . . . 18

3.2.1 Channel Estimate Improvement . . . . . . . . . . . . 18

3.2 .2 Random Access . . . . . . . . . . . . . . . . . . . 19

3.2 .3 Coverage ................... 20

4 Future Work 23

$\begin{array}{ll}\text { Bibliography } & 24\end{array}$ 
A Joint Antenna Detection and Channel Estimation 37

1 Introduction . . . . . . . . . . . . . . . 39

1.1 Contributions . . . . . . . . . . . . . 41

1.2 Notation . . . . . . . . . . . . . . . . . 42

2 System Model . . . . . . . . . . . . . . . . . . 42

3 Channel Estimation . . . . . . . . . . . . . . . . 43

4 Single Antenna Terminal with Phase Shifts . . . . . . . . . 44

$4.1 \quad$ Solutions . . . . . . . . . . . . . . . . . 48

$5 \quad$ Antenna Detection . . . . . . . . . . . . . 51

$5.1 \quad$ K-means Clustering . . . . . . . . . . . . 52

5.2 Expectation-Maximization (EM) Algorithm . . . . . . 52

$5.3 \quad$ Spectral Clustering . . . . . . . . . . . . . . 53

6 Joint Antenna Detection and Channel Estimation . . . . . . . 55

7 Acheivable Rate . . . . . . . . . . . . . . . . . 56

8 Numerical Simulations . . . . . . . . . . . . . . . . . 60

9 Conclusions . . . . . . . . . . . . . . . 68

B Detection of Pilot-Hopping Sequences $\quad 73$

1 Introduction . . . . . . . . . . . . . . 75

1.1 Related Works . . . . . . . . . . . . 76

1.2 Contributions . . . . . . . . . . . . 76

2 System Model . . . . . . . . . . . . . . . . . . 77

3 Detecting Active Users Using the Asymptotic Energies . . . . 78

4 Maximum Likelihood Detection of Active Users . . . . . . . . 80

5 Maximum a Posteriori Detection of Active Users . . . . . . . 81

6 Simulations . . . . . . . . . . . . . . . 82

7 Conclusions . . . . . . . . . . . . . . 85

C How Much Will Tiny IoT Nodes Profit from Massive MIMO? 89

1 Introduction . . . . . . . . . . . . . . 91

$1.1 \quad$ Notation . . . . . . . . . . . . . . . . 93

1.2 Background on Current IoT Solutions . . . . . . . . 93

2 System Model . . . . . . . . . . . . . . . . . . . . . 94

3 Power Scaling . . . . . . . . . . . . . . . . . . . . 97 97

4 Simulations . . . . . . . . . . . . . . . . . . . 98

5 Conclusion .................... . 100

Appendix A: Proof of Upper Bound in Lemma 1 . . . . . . . . 101 


\section{Acknowledgements}

First of all, I'm very grateful to my supervisors Professor Erik G. Larsson and Associate Professor Emil Björnson. Thank you for leading by example and inspiring me with your dedication to research and knowledge. You guidance helps me evolve and improve in the right direction.

To all my colleagues, thank you for creating a great working environment. I feel like I can thrive in your presence. I appreciate all the discussions we have, both the work related ones and the more casual, it makes going to the office very enjoyable!

To all my cool and awesome friends, thank you! I always have a great time hanging out with you. You always know exactly how much support I need and if when I need to rant. You make me happy and I feel like I can relax in your company.

Finally, a big thanks to my family who never doubt or question me, with your support I feel like I can do anything!

Ema Becirovic

Linköping, January 2020 


\section{List of Abbreviations}

$5 \mathrm{G}$

fifth generation of cellular network technology

AWGN additive white Gaussian noise

DL

downlink

eMBB

enhanced mobile broadband

FDD

frequency-division duplex

i.i.d.

independent and identically distributed

IoT

internet-of-things

LoS

line-of-sight

LPWA low-power wide-area

LS least-squares

MIMO

multiple-input multiple-output

MMSE

minimum mean-square error

mMTC

massive machine-type communication

$\mathrm{MR}$

maximum ratio

NLoS

non line-of-sight

$\mathrm{RF}$

radio frequency

SNR

signal-to-noise ratio

TDD

time-division duplex

UL uplink

URLLC ultra-reliable low-latency communication $\mathrm{ZF}$ zero forcing 



\section{Chapter 1}

\section{Introduction and Motivation}

Wireless communication is always evolving and changing. We have seen everything from rudimentary cellular systems such as $2 \mathrm{G}$ where the focus was on phone calls to high-speed Wi-Fi commonly used for wireless Internet access both in private homes and public spaces. Therefore, one may ask themselves: what is next? Well, in terms of cellular communication, the answer is the fifth generation of cellular network technology (5G).

A communication system is like a game where one has to sacrifice an attribute in order to gain another. As an example, an increase of transmit power gives an increase in signal-to-noise ratio (SNR) but decreases the battery lifetime of a device. Therefore, going into the future, wireless communication has been split into use-cases, where each use-case focuses on a specific scenario with a set of requirements [1]. The use-cases envisioned for the future wireless communication are the trifecta: enhanced mobile broadband (eMBB), ultra-reliable low-latency communication (URLLC), and massive machine-type communication (mMTC) [2]. The three use-cases cover different requirements and needs of the future:

eMBB is intended to give higher data rates to be used for applications such as virtual reality, augmented reality, and high quality video streaming, amongst other demanding applications.

URLLC is intended for time-sensitive communication such as communication between vehicles [3], remote surgery [3,4], and envisioned to essentially replace cables in industrial settings for robots and similar $[3,5,6]$. These applications need high reliability and low latency, but the data rates need not be very high.

mMTC is intended to cope with the massive increase of low data rate devices 
such as different types of sensors $[7,8]$. While there are many of them, the individual devices do not transmit large amounts of data, they are not time-critical, and they can be assumed to have low mobility.

Together these three use-cases cover the anticipated needs of wireless communication for both humans and machines in the coming years.

One of the technologies that will help fulfill the use-case requirements is massive multiple-input multiple-output (MIMO). Massive MIMO is a technology that has been established as one of the most important technologies that makes 5G possible [9]. Since it was conceived in [10], it has been prevalent in communications research. Massive MIMO is essentially a communication system, operating in time-division duplex (TDD) mode, where a base station with a massive number of antennas serves multiple users in the same timeand frequency resource by spatially multiplexing them. ${ }^{1}$ The technology then relies on the law of large numbers [12] to produce elegant mathematical expressions which simplifies the signal processing. Importantly, massive MIMO technology has been empirically shown to work with the help of test beds in both academia and in industry [13]. It is also currently being deployed as a $5 \mathrm{G}$ technology. The specifics of massive MIMO will be discussed further in Chapter 2.

The main focus of massive MIMO research has been increasing the data rates, i.e., targeted towards the eMBB requirements. It has been very successful in this aspect and can increase the spectral efficiency, i.e., the number of bits that can be transmitted per second in a given frequency band and area [14]. This is achieved by being able to spatially multiplex users in the same time- and frequency resource. To increase the spectral efficiency several aspects might be considered. One aspect that is important is power control [14-23]. The power control can aim to create fairness between the users or increase the sum spectral efficiency in the network. Many more aspects of massive MIMO have be considered, such as pilot assignment [22, 24-27], broadcast and multicast [28-33], physical-layer security [34-40], and hardware impairments [41-51]. A massive MIMO related technology that has been on the rise lately is cell-free massive MIMO [52-56]. It erases the cell boundaries by serving one user with multiple single-antenna base stations called access points. Cell-free massive MIMO gives benefits by decreasing the probability that a user has a bad channel to the access points.

Massive MIMO is not only beneficial for the eMBB use-case - it also provides some benefits for URLLC $[57,58]$. Using massive MIMO can help reduce the outage probability, and therefore increase the reliability, compared

\footnotetext{
${ }^{1}$ See [11] for a discussion on the definition of massive MIMO.
} 
to a system with single-antenna base stations. Due to the increased reliability, there is less need for retransmitting packets, which means that the latency will also reduce. Additionally, the latency for a user can further be improved as the user does not have to wait for other users to be served until it itself can be served. This is due to the base station being able to serve multiple users in the same time- and frequency block. A note of caution for URLLC in massive MIMO systems is that massive MIMO conventionally operates in TDD which delays the communication, and hence increasing latency. Another metric, related to latency, is age of information [59]. Age of information is (a function of) the difference in time between the last received packet and when it was generated. This metric is closely related to latency but is more suitable when considering the cases where the data is related between different transmissions.

In addition to massive MIMO being beneficial for eMBB and URLLC, massive MIMO is also beneficial for the mMTC use-case [58,60,61]. The most straight-forward benefit is the increase in SNR due to the beamforming gain [15]. With higher SNR, wireless communication with remote sensors and other mMTC devices will be possible since higher attenuations can be tolerated. The increased SNR can also be used to lower the transmit power of the devices and therefore increase the battery life of the devices [62]. Another benefit is that it is easier to separate users in massive MIMO [14]. This helps the base station detect users in the system's random access phase [63]. For mMTC, it is preferable if the random access is grant-free, meaning that the users send their identification and data without waiting for an acknowledgment from the base station [64-66].

The users in a network are not homogeneous. They have different requirements and are in different use-cases. We usually study the use-cases in isolation - in the paragraphs above use-cases were considered individually. Nonetheless, it is important to show that the users of the different use-cases can coexist $[67,68]$ — which they can.

\subsection{Contributions of the Thesis}

This thesis considers three research problems related to mMTC, and how these problems can be solved by using massive MIMO technology. The thesis is split in two parts; the first part gives an introduction and some preliminaries of the topics covered in the thesis and the second part is a collection of papers which consider specific problem formulations occurring in mMTC. More specifically, Paper A considers how channel estimates for 
stationary, or low mobility, users can be improved. Paper B proposes a solution for grant-free random access of a massive number of mMTC users. Finally, Paper $\mathrm{C}$ considers the benefits of massive MIMO for low-power wide-area mMTC users.

\section{Paper A: Joint Antenna Detection and Bayesian Channel Estimation for Non-Coherent User Terminals}

Authored by: Ema Becirovic, Emil Björnson, and Erik G. Larsson

Paper submitted to IEEE Transactions on Wireless Communications.

Abstract: In this paper, we propose a method of improving the channel estimates for non-coherent multi-antenna terminals, which are terminals that cannot control the relative phase between its antenna ports, with channels that can be considered constant over multiple time slots. The terminals have multiple antennas and are free to choose whichever antenna they want to use in each time slot. An unknown phase shift is introduced in each time slot as we cannot guarantee that the terminals are phase coherent across time slots. We compare three different clustering techniques that we use to detect the active antenna. We also compare a set of different statistical and heuristic estimators for the channels and the phase shifts. We evaluate the methods by using correlated Rayleigh fading and three different bounds on the uplink capacity. The accuracy of the capacity bounds are verified with bit-error-rate simulations. With our proposed methods we can have an SNR improvement of approximately $2 \mathrm{~dB}$ at $1 \mathrm{bit} / \mathrm{s} / \mathrm{Hz}$.

\section{Paper B: Detection of Pilot-Hopping Sequences for Grant-Free Random Access in Massive MIMO Systems}

Authored by: Ema Becirovic, Emil Björnson, and Erik G. Larsson

Published in the proceedings of 2019 IEEE International Conference on Acoustics, Speech, and Signal Processing (ICASSP)

Abstract: In this paper, we study an active user detection problem for massive machine type communications (mMTC). The users transmit pilothopping sequences and detection of active users is performed based on the received energy. We utilize the channel hardening and favorable propagation properties of massive multiple-input multiple-output (MIMO) to simplify the user detection. We propose and compare a number of different user detection methods and find that using non-negative least squares (NNLS) is well suited for the task at hand as it achieves good results as well as having the benefit of not having to specify further parameters. 


\section{Paper C: How Much Will Tiny IoT Nodes Profit from Massive Base Station Arrays?}

Authored by: Ema Becirovic, Emil Björnson, and Erik G. Larsson

Published in the proceedings of 2018 26th European Signal Processing Conference (EUSIPCO)

Abstract: In this paper we study the benefits that Internet-of-Things (IoT) devices will have from connecting to a massive multiple-input-multiple-output (MIMO) base station. In particular, we study how many users that could be simultaneously spatially multiplexed and how much the range can be increased by deploying massive base station arrays. We also investigate how the devices can scale down their uplink power as the number of antennas grows with retained rates.

We consider the uplink and utilize upper and lower bounds on known achievable rate expressions to study the effects of the massive arrays. We conduct a case study where we use simulations in the settings of existing IoT systems to draw realistic conclusions.

We find that the gains which ultra narrowband systems get from utilizing massive MIMO are limited by the bandwidth and therefore those systems will not be able to spatially multiplex any significant number of users. We also conclude that the power scaling is highly dependent on the nominal signal-to-noise ratio (SNR) in the single-antenna case.

\subsection{Excluded Papers}

The papers in Table 1 are coauthored by me but excluded from the thesis due to them being superfluous. The first paper is a conference version of Paper A and the second paper presents an architecture for detecting pilot-hopping sequences based on Paper B. 
E. Becirovic, E. Björnson, and E. G. Larsson, "Joint Antenna Detection and Channel Estimation for Non-Coherent User Terminals," in 2019 IEEE 20th International Workshop on Signal Processing Advances in Wireless Communications (SPAWC), Cannes, France, 2019, pp. 1-5.

N. Mohammadi Sarband, E. Becirovic, M. Krysander, E. G. Larsson, and O. Gustafsson, "Pilot-Hopping Sequence Detection Architecture for GrantFree Random Access using Massive MIMO," Accepted to the IEEE International Symposium on Circuits and Systems (ISCAS) 2020. 


\section{Chapter 2}

\section{Massive MIMO}

In this chapter the conventional single-cell massive MIMO system is presented and discussed. The chapter serves to remind the reader about the benefits of massive MIMO that are not directly connected to the mMTC use-case.

\subsection{System Model}

We assume a simple, single-cell massive MIMO system. The base station has $M$ antennas, and serves $K$ single-antenna users ${ }^{1}$. We study this system in a sufficiently small enough time-frequency block, called the coherence block, such that we can consider the channel between any base-station antenna and any user to be constant. This is called the block-fading model. The size of this block is further discussed in Section 2.1.2.

Each of the base-station antennas has one radio frequency (RF) chain such that the base station can digitally control the relative phase between all of its antenna elements and which therefore makes the base station capable of spatially directing signals to the users [14].

The channel between a base-station antenna $m$ and a user $k$, which encompasses multipath propagation (i.e., scattering, diffusion, and reflection $[69,70])$, attenuation, and shadowing, can be characterized by two complex ${ }^{2}$ values, one for the case when the base-station antenna is transmitting and the user is receiving - the downlink (DL), and one for the case when the user is transmitting and the base-station antenna is receiving - the uplink (UL).

\footnotetext{
${ }^{1}$ Throughout the thesis, the users will be called users, devices, terminals and sensors, interchangeably.

${ }^{2}$ The channel is not actually complex, but is a notational consequence of using complex baseband representation as is common in communication $[71,72]$.
} 


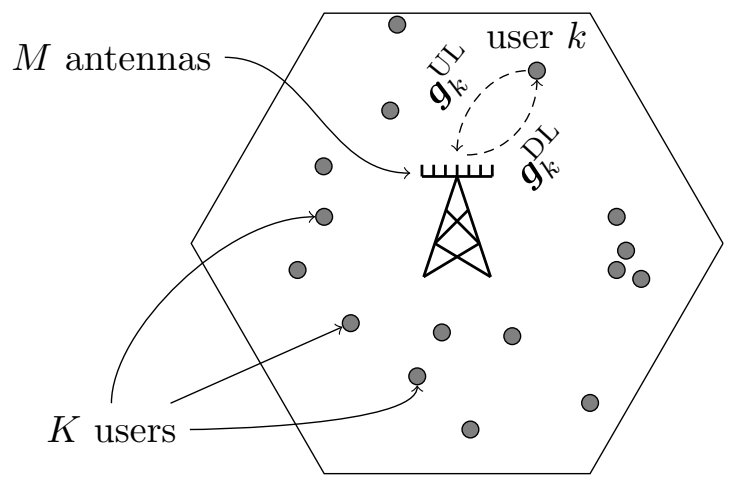

Figure 1: A small illustration depicting the single-cell massive MIMO system model. The base station is equipped with $M$ antennas and serves $K$ single-antenna users. The UL and DL channel to user $k$ is denoted by $\boldsymbol{g}_{k}^{\mathrm{UL}}$ and $\boldsymbol{g}_{k}^{\mathrm{DL}}$, respectively.

We group these values in vectors and denote the UL and DL channels of a user $k$ by $\boldsymbol{g}_{k}^{\mathrm{UL}} \in \mathbb{C}^{M}$ and $\boldsymbol{g}_{k}^{\mathrm{DL}} \in \mathbb{C}^{M}$, respectively. Figure 1 shows a small illustration of the system model.

We are assuming that we are operating in TDD, i.e., that transmission in the UL and DL are separated in time, as opposed to frequency-division duplex (FDD), where the UL and DL are separated in frequency. The reason for TDD operation will become apparent after the discussion about reciprocity in Section 2.1.1. Finally, the received signals are affected by additive white Gaussian noise (AWGN).

\subsubsection{Reciprocity}

One key assumption that helps cement massive MIMO as a key technology of $5 \mathrm{G}$ is the assumption of reciprocity. This means that the UL and DL channel of a user and base-station antenna pair are the same. Hence, we drop the superscripts and refer to the channel of user $k$ as

$$
\boldsymbol{g}_{k}=\boldsymbol{g}_{k}^{\mathrm{UL}}=\boldsymbol{g}_{k}^{\mathrm{DL}}
$$

regardless of whether the user is transmitting or receiving. Usually, to simplify the notation, we consider the DL channel to be the conjugate (hermitian) transpose of the UL channel without loss of generality. The channels of the users are grouped in the matrix $\boldsymbol{G}=\left[\boldsymbol{g}_{1}, \ldots, \boldsymbol{g}_{K}\right]$, for notational purposes.

With the reciprocity assumption, we only need to estimate the channel of a user in one direction. This is very important for massive MIMO because if 
we had to estimate the DL channel, the time spent on training would increase with the number of base-station antennas, $M[14,15]$.

Note that the physical propagation environment is always reciprocal when TDD is used. The reason why reciprocity is not always assumed is because the transmit and receive RF chains are not generally reciprocal [73]. However, there exists methods of reciprocity calibrating the RF chains, e.g., see [74], hence, the reciprocity assumption is reasonable.

\subsubsection{Coherence Block}

By the block-fading channel assumption we assume that the channel is constant in a time-frequency interval called the coherence block or the coherence interval. The coherence block is the product of two intervals, the coherence time, which is the time interval where we can consider the channel to be time invariant, and the coherence bandwidth, which is the frequency interval where we can consider the channel to be flat $[14,15,71]$.

The coherence time, $T_{\mathrm{c}}$, of a channel depends on the Doppler spread of the channel, which in turn is proportional to the wavelength and inversely proportional to the velocity of the transmitter $[15,71]$. This means that a user on a moving train will have much lower coherence time than a user that is standing still.

The coherence bandwidth, $B_{\mathrm{c}}$ of a channel will depend on the delay spread of the channel which in turn depends on the difference between the shortest and longest paths in the propagation environment [15,71].

The size of the coherence block is a product of the coherence time and the coherence bandwidth, $\tau_{\mathrm{c}}=T_{\mathrm{c}} B_{\mathrm{c}}$, and is different for each user. Because we need a system wide coherence block, we refer to the coherence block as the smallest coherence block of all the users. The size of the coherence block is usually on the order of hundreds of symbols. However, the size can increase to several thousands if the users are stationary $[15,70]$.

\subsubsection{Channel Model}

We use channel models as mathematical and simulation tools to e.g., construct estimators and evaluate performance, as we cannot perform experiments in real world systems that have not been constructed yet. The most tractable channel model is a case where there is no multipath propagation. We call this a line-of-sight (LoS) channel. In the LoS channel model, the transmitter and receiver are assumed to be in free space. Only the distance between the two will affect the channel $[15,70]$. The LoS channel model is also used when we 
have a strong LoS component and the non line-of-sight (NLoS) components have significantly smaller, negligible, power.

As soon as we start mixing in multipath components we will have a more complicated model. If the number of paths which the signal takes is only a handful, we can try to model all the paths with a deterministic ray-tracing model $[75,76]$. However, this approach quickly becomes intractable and hard to analyze.

In a multipath channel, several attenuated and time-delayed copies of a transmitted signal will arrive at the receiver. As the channel is constantly changing due to objects that move around in the propagation environment, we cannot feasibly track all the time delays and attenuations from multipaths in the channel, hence it is common to employ a statistical model of the channel. The statistics of the channel will be constant over a time that is longer than the coherence time. Hence, they will have to be estimated but can be done more infrequently than estimating the instantaneous channel.

One of the most common channel model assumptions, which is also the one used in this thesis, is the Rayleigh fading channel which is based on the central limit theorem [12], i.e., that the addition of many paths with different statistical distributions will approach a normal distribution [14,71]. The Rayleigh fading channel gets its name from that the channel induces an amplitude that follows a Rayleigh distribution $[69,70]$.

In the Rayleigh fading model the channel in each coherence block follows a circularly symmetric complex Gaussian distribution, so for a user $k$, its channel is distributed as

$$
\boldsymbol{g}_{k} \sim \mathcal{C N}\left(\mathbf{0}, \boldsymbol{R}_{k}\right),
$$

where $\boldsymbol{R}_{k}$ is the (spatial) correlation matrix of user $k$. If there is no spatial correlation the channel is said to be uncorrelated Rayleigh fading and the correlation matrix is equal to a scaled identity matrix, $\boldsymbol{R}=\beta_{k} \boldsymbol{I}$. The largescale fading coefficient, $\beta_{k}$, captures the attenuation of the $k$ :th channel and depends on the distance between the user and the base station. In the case of correlated Rayleigh fading, there are several different ways of modeling the correlation. Often it is assumed that there is a group of scatterers concentrated in one or more locations. This assumption leads to models such as the local scattering spatial correlation model $[14,70]$. The large-scale fading coefficient and the spatial correlation matrix can be estimated with different methods [77-80].

Of course, there are many more different statistical channel models to employ in massive MIMO. Examples of these are the Rician fading channel $[70,81]$, and the keyhole channel $[15,70]$. 
With some channel models, including the uncorrelated Rayleigh fading channel model, we get two important properties which will make dealing with a massive number of base-station antennas easier. The first property is called channel hardening and is mathematically defined as $[14,15,70]$

$$
\frac{\left\|\boldsymbol{g}_{k}\right\|^{2}}{\mathbb{E}\left\{\left\|\boldsymbol{g}_{k}\right\|^{2}\right\}} \stackrel{\text { a.s. }}{\rightarrow} 1, \quad \text { as } M \rightarrow \infty,
$$

for a channel $\boldsymbol{g}_{k} \in \mathbb{C}^{M}$, with $M$ antennas. In layman's terms, the norm of a channel that has the channel hardening property will act deterministically if the number of the antennas is sufficiently large. This is a property that helps with signal processing and deriving closed-form bounds on the channel capacity.

The other property is called asymptotic favorable propagation and its definition is $[14,15,70]$

$$
\frac{\boldsymbol{g}_{k}^{\mathrm{H}} \boldsymbol{g}_{k^{\prime}}}{\sqrt{\mathbb{E}\left\{\left\|\boldsymbol{g}_{k}\right\|^{2}\right\} \mathbb{E}\left\{\left\|\boldsymbol{g}_{k^{\prime}}\right\|^{2}\right\}}} \stackrel{\text { a.s. }}{\rightarrow} 0, \quad \text { as } M \rightarrow \infty,
$$

where $\boldsymbol{g}_{k} \in \mathbb{C}^{M}$ and $\boldsymbol{g}_{k^{\prime}} \in \mathbb{C}^{M}$ denote the channels of two different users, $k \neq k^{\prime}$. This property is more simply stated as: if we have sufficient number of antennas the channels of two different users will be almost orthogonal. This helps separate users such that they can be served in the same time- and frequency block.

\subsection{Channel Estimation}

To be able to effectively direct the signals to the users we need to estimate the channel. Because we have assumed reciprocity it suffices to estimate the channel in one direction. In massive MIMO, due to having more base-station antennas than users, we estimate the UL channel. Therefore, the coherence block of a conventional massive MIMO system is split into three parts: the UL training phase, the UL data phase, and the DL data phase, as pictured in Figure $2[14,15]$. The training phase can be placed anywhere in the coherence block as long as it placed before the DL data phase. In fact, one can also superimpose the UL data and the training symbols [82,83], however this is not covered in conventional massive MIMO. By studying Figure 2, one notices that the more symbols we spend on training, the less time we have to transmit data. Thus, while increasing the number of symbols used for training improves the channel estimates, the rate might go down as the number of transmitted information bearing bits is smaller. 


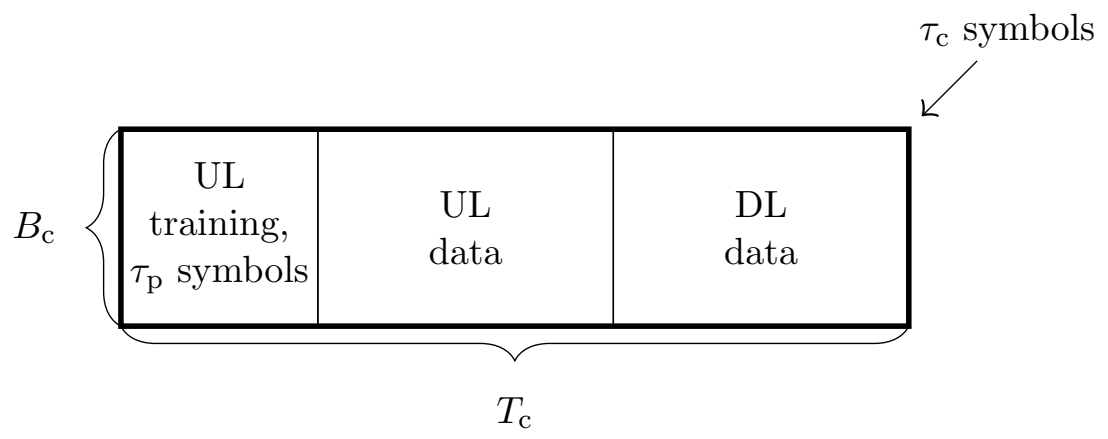

Figure 2: A depiction of the massive MIMO coherence block. The coherence block spans $\tau_{\mathrm{c}}=T_{\mathrm{c}} B_{\mathrm{c}}$ symbols of which $\tau_{\mathrm{p}}$ are used for training. The UL and DL data transmission parts are divided in time.

To help us estimate the channels, we start by assigning a sequence of symbols to each user. This sequence is called a pilot and the pilot of user $k$ is denoted as $\phi_{k} \in \mathbb{C}^{\tau_{\mathrm{p}}}$. The pilots assigned to the different users are mutually orthogonal, $\boldsymbol{\phi}_{k}^{\mathrm{H}} \phi_{k^{\prime}}=0, k \neq k^{\prime}$, have unit energy, $\left\|\phi_{k}\right\|=1, k=1, \ldots, K$, and have length $\tau_{\mathrm{p}}$. Hence, $K \leq \tau_{\mathrm{p}}<\tau_{\mathrm{c}}$. We group the pilots in the matrix $\boldsymbol{\Phi}=\left[\phi_{1}, \ldots, \phi_{K}\right]$, for notational purposes. If the pilots are not mutually orthogonal, a phenomenon called pilot contamination occurs and the users cannot be separated. Pilot contamination can in some cases be very degrading for the performance of massive MIMO $[10,14,84]$. All the $K$ users transmit their pilot ${ }^{3}$ over their respective channels at the same time and the base station receives

$$
\boldsymbol{Y}^{\mathrm{p}}=\sqrt{\rho_{\mathrm{ul}} \tau_{\mathrm{p}}} \boldsymbol{G} \boldsymbol{\Phi}^{\mathrm{H}}+\boldsymbol{W}^{\mathrm{p}},
$$

where $\rho_{\mathrm{ul}}$ is the normalized UL SNR per pilot symbol and $\boldsymbol{W}^{\mathrm{p}} \in \mathbb{C}^{M \times \tau_{\mathrm{p}}}$ is AWGN with independent and identically distributed (i.i.d.) $\mathcal{C N}(0,1)$ elements. A sufficient statistic of the channel of user $k$ is

$$
\boldsymbol{y}_{k}^{\mathrm{p}}=\boldsymbol{Y}^{\mathrm{p}} \boldsymbol{\phi}_{k}=\sqrt{\rho_{\mathrm{ul}} \tau_{\mathrm{p}}} \boldsymbol{g}_{k}+\boldsymbol{w}_{k}^{\mathrm{p}},
$$

where $\boldsymbol{w}_{k}^{\mathrm{p}}=\boldsymbol{W}^{\mathrm{p}} \boldsymbol{\phi}_{k} \sim \mathcal{C N}(\mathbf{0}, \boldsymbol{I})$.

We assume that we know that the channels are uncorrelated Rayleigh fading and that we know all the large-scale fading coefficients of all the users. The best we can produce is the minimum mean-square error (MMSE) estimator, which for user $k$ is $[15,85]$

$$
\hat{\boldsymbol{g}}_{k}=\frac{\sqrt{\rho_{\mathrm{ul}} \tau_{\mathrm{p}}} \beta_{k}}{1+\rho_{\mathrm{ul}} \tau_{\mathrm{p}} \beta_{k}} \boldsymbol{y}_{k}^{\mathrm{p}} .
$$

\footnotetext{
${ }^{3}$ In (5) the users are transmitting the conjugate of the pilots to simplify notation.
} 
The mean-square of the channel estimate between base-station antenna $m$ and user $k$ is $[15,85]$

$$
\gamma_{k}=\mathbb{E}\left\{\left|\left[\hat{\boldsymbol{g}}_{k}\right]_{m}\right|^{2}\right\}=\frac{\tau_{\mathrm{p}} \rho_{\mathrm{ul}} \beta_{k}^{2}}{1+\tau_{\mathrm{p}} \rho_{\mathrm{ul}} \beta_{k}} .
$$

When the channel estimate is good, the mean-square of the channel estimate will be close to the large-scale fading coefficient.

Other, inferior, estimators exist. One of which is the least-squares (LS) estimator which does not consider any knowledge of the channel. The LS estimator is $[14,85]$

$$
\hat{\boldsymbol{g}}_{k}=\frac{\boldsymbol{y}_{k}^{\mathrm{p}}}{\sqrt{\rho_{\mathrm{ul}} \tau_{\mathrm{p}}}} .
$$

A disadvantage of the LS estimator is that it will generally not give an estimate that is uncorrelated with the estimation error, which is not the case for the MMSE estimator [14].

\subsection{Spatial Multiplexing}

The magic of massive MIMO comes from that the base station can spatially multiplex users by directing signals using something called beamforming. What is done in the DL case is that the base station uses the channel estimates of the users and tries to come up with precoders that will constructively or destructively interfere at the position of the users' antennas. In the UL this process is called receive combining wherein the base station combines the received signals from each antenna with a phase shift and an amplitude scaling.

To make it more clear, we illustrate this process with a small example. We study a case where a single antenna transmitter sends a sinusoid to a two-antenna receiver. In this example, we omit the effects of the channel. When decoding the transmitted sinusoid, the receiver can choose to add the received sinusoids coherently, and the resulting signal will have a higher amplitude (SNR) than if the received signals are considered individually. Similarly, the receiver can phase shift one of the received signals by half of a period, which will make the two signals cancel out.

These two cases are directly translatable to the receive combining vectors or linear decoders ${ }^{4}$. The case where the aim is to maximize the SNR at the

\footnotetext{
${ }^{4}$ We do not state the precoders here as the thesis will mainly focus on UL transmission.
} 
user is called maximum ratio (MR) [15] and equates to the receive combining vectors (matrix) of the users being:

$$
\boldsymbol{V}^{\mathrm{MR}, \mathrm{UL}}=\hat{\boldsymbol{G}}
$$

where $\hat{\boldsymbol{G}}=\left[\hat{\boldsymbol{g}}_{1}, \ldots, \hat{\boldsymbol{g}}_{K}\right]$. That is, the $k$ :th column of $\boldsymbol{V}^{\mathrm{MR}, \mathrm{UL}}$ corresponds to the receive combining vector of user $k$ which in this case is the channel estimate of user $k, \hat{\boldsymbol{g}}_{k}$.

In the case that is called zero forcing (ZF) [15] the base station tries to cancel out all the signals from the other users when decoding the intended user. In the ideal case, where the channel is perfectly known there will be no interference from the signals intended to the other users at all. The receive combining vectors for the $\mathrm{ZF}$ case is:

$$
V^{\mathrm{ZF}, \mathrm{UL}}=\hat{\boldsymbol{G}}\left(\hat{G}^{\mathrm{H}} \hat{\boldsymbol{G}}\right)^{-1} .
$$

Ergo, the ZF receive combing vector for a user $k$ corresponds to projecting the signal onto the null-space of all other channels. Hence, ZF can only be used if there are more antennas at the base station than there are users [15].

\subsection{Capacity Bounds}

One of the most important ways to measure the performance of a communication system is to look at the capacity. The capacity is the maximum amount of information that can be sent over a channel with an arbitrarily small error [71].

It is in many cases very hard to find an expression for the channel capacity. We can calculate the channel capacity for simple channels such as the AWGN channel or a channel with deterministic fading [71]. However, what we usually rely on are lower bounds on the channel capacity. The lower bounds are often times referred to as achievable rates because we can actually achieve the rates that they are predicting!

One lower bound on the capacity is zero, however this bound is extremely silly and does not give us any insight about the performance of the system. Perhaps one of the best bounds used is the side-information bound $[14,15]$. The side-information refers to that the receiver has some implicit knowledge about the channel, such as the channel estimate, but not necessarily the channel itself. The UL achievable rate predicted by the side-information bound for user $k$ in a massive MIMO system with uncorrelated Rayleigh 
fading and MMSE channel estimation is [15]

$$
C_{k} \geq \mathbb{E}\left\{\log _{2}\left(1+\frac{\rho_{\mathrm{ul}} \eta_{k}\left|\boldsymbol{v}_{k}^{\mathrm{H}} \hat{\boldsymbol{g}}_{k}\right|^{2}}{\sum_{\substack{k^{\prime}=1 \\ k^{\prime} \neq k}}^{K} \rho_{\mathrm{ul}} \eta_{k^{\prime}}\left|\boldsymbol{v}_{k}^{\mathrm{H}} \hat{\boldsymbol{g}}_{k^{\prime}}\right|^{2}+\left\|\boldsymbol{v}_{k}\right\|^{2} \sum_{k^{\prime}=1}^{K} \rho_{\mathrm{ul}} \eta_{k^{\prime}}\left(\beta_{k^{\prime}}-\gamma_{k^{\prime}}\right)+\left\|\boldsymbol{v}_{k}\right\|^{2}}\right)\right\},
$$

where $\eta_{k}$ is the power-control coefficient of user $k$, i.e., a scalar between 0 and 1 which represents how much of the total allowed power that the user should spend. The expectation is with respect to the side-information, i.e., the channel estimates. Generally, we cannot find a closed-form expression for this expectation and it is hard to use this bound to construct meaningful signal processing algorithms, such as power-control schemes.

Because of this reason, we often use another bound - the use-and-forget bound $[14,15]$. The name refers to that we use the knowledge about the channel estimates in the receive combining, but then throw the knowledge away when detecting the signal. The UL achievable rate predicted by the use-and-forget bound for user $k$ in a massive MIMO system with uncorrelated Rayleigh fading and MMSE channel estimation is [15]

$C_{k} \geq \log _{2}\left(1+\frac{\rho_{\mathrm{ul}} \eta_{k}\left|\mathbb{E}\left\{\boldsymbol{v}_{k}^{\mathrm{H}} \boldsymbol{g}_{k}\right\}\right|^{2}}{\sum_{k^{\prime}=1}^{K} \rho_{\mathrm{ul}} \eta_{k^{\prime}} \mathbb{E}\left\{\left|\boldsymbol{v}_{k}^{\mathrm{H}} \boldsymbol{g}_{k^{\prime}}\right|^{2}\right\}-\rho_{\mathrm{ul}} \eta_{k}\left|\mathbb{E}\left\{\boldsymbol{v}_{k}^{\mathrm{H}} \boldsymbol{g}_{k}\right\}\right|^{2}+\mathbb{E}\left\{\left\|\boldsymbol{v}_{k}\right\|^{2}\right\}}\right)$.

By utilizing the use-and-forget bound we can perform very profitable power control that can help increase the fairness among the users or the overall spectral efficiency of the whole system [15]. This is actually one of the benefits of massive MIMO; we only need to use the long term channel statistics in our power control optimization problems. Hence, the power control can be performed more sparsely - only when the large-scale fading of the channel has changed. 


\section{Chapter 3}

\section{Massive Machine-Type Communications}

In this chapter we discuss the assumptions in mMTC and what problems that arise. Further, we demonstrate how massive MIMO can solve some of these problems.

\subsection{Assumptions}

The mMTC use-case is intended for the ever increasing number of smart devices. We describe mMTC by a set of requirements and assumptions. These are $[2,58]$ :

- lenient latency requirements,

- a huge number of devices,

- low transmit power of the devices,

- predominantly UL communication,

- low per-user data rates,

- wide area coverage, and

- low mobility of devices.

It can be noted that mMTC is a subset of what is called internet-of-things (IoT), which has become quite a buzzword. However, the hype is justified - 
it is predicted that the number of cellular IoT devices will be 4.1 billion in $2024[86]$ !

The mMTC use-case is designed to handle sensor networks in homes [87,88], smart-cities [89], and industrial scenarios [90]. We imagine a city where all the electricity meters are connected to the cloud through a set of base stations. Clearly, the measurements produced by the electricity meters are not time critical; it does not matter if it takes a couple of seconds for the measurements to reach the cloud. The number of electricity meters is huge, every household has one. Also, not all houses are close to a base station. The devices transmit in the UL as they do not have any other purpose than to monitor the electricity and the data rates are low as each transmission only sends a few sensor measurements. The only requirement or assumption that this example did not fulfill is the energy efficiency requirement. This is because it is nonsensical to assume that a sensor which is measuring electricity is not connected to the electrical grid. However, one can envision other sensors that run on batteries and which might be placed in inconvenient locations such that changing the batteries frequently is unsuitable.

\subsection{Massive MIMO for mMTC}

In this section we highlight some of the improvements that massive MIMO provides when applied to mMTC. We stress that these are only a subset of the benefits that massive MIMO gives when applied to mMTC.

\subsubsection{Channel Estimate Improvement}

Cellular systems are designed to accommodate users with high mobility and high data rate, such as people sitting on the train while streaming a high definition video. The size of the coherence block is designed with this use-case in mind. However, there might be a sensor somewhere in the system which is not moving at all. It is only the mobility of the surrounding environment that will affect the coherence block of this sensor. Because the system is designed for the high mobility user, the sensor, with a coherence block much larger than the high mobility user, will effectively estimate the same channel multiple times, see Figure 3.

The base station can exploit the knowledge of the sensor being stationary and combine the estimates in consecutive time slots to improve the overall channel estimate. This superior channel estimate can then be used to decode data from the sensor. We assume that the sensor is not receiving DL data and that it does not have any latency requirements, hence, we can wait to 
Coherence block of high mobility user

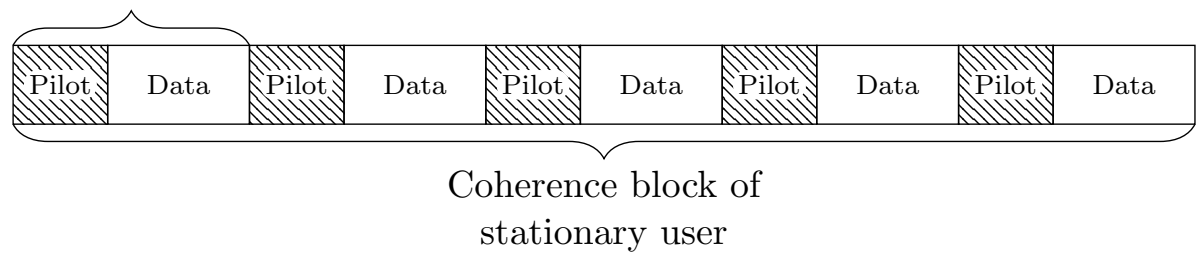

Figure 3: A high mobility user has a much shorter coherence block than a stationary user. As a consequence, the stationary user will estimate the same channel in many consecutive time slots.

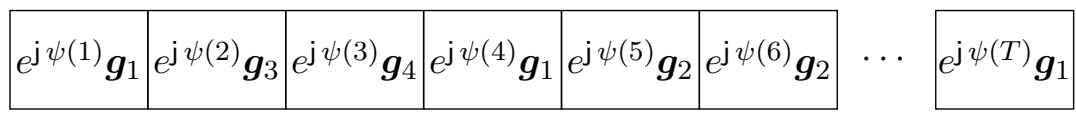

Figure 4: The user depicted in this figure has four antennas with channels $\boldsymbol{g}_{1}, \boldsymbol{g}_{2}$, $\boldsymbol{g}_{3}$ and $\boldsymbol{g}_{4}$ which are constant for $T$ slots. Even though the physical channel is constant for $T$ slots, the effective channel might change due to unknown phase shifts, $\psi(t), t=1, \ldots, T$, or antenna switching.

decode the UL data.

Nevertheless, the system is still designed with the high mobility user in mind which means that the requirements put on the users is designed for the high mobility case. What might happen, due to a lack of specification in standards and sub-optimal hardware is that the sensor will introduce a phase shift or switch antennas in each time slot, see Figure 4. This is not a problem in the conventional case as the users are reestimating their channels in each slot. However, it will need to be compensated for to utilize the channel estimate improvements. This is the scenario and problem formulation studied in Paper A.

\subsubsection{Random Access}

Random access is the process where the users get access to the base station [91]. It is a bottleneck of mMTC due to the vast number of users present. The random access procedure can be grant-free or not. If the random access is not grant-free, users that want to connect to the network first send a sequence of symbols. The base station receives these sequences and decides, for each user, if the user should get access to the network or if it should try again. The base station then sends this information to the users. If the random 
access is grant-free, there is no response from the base station. The users will send their identifying sequence and data, cross their fingers and hope that the base station successfully received it.

As mentioned in Section 3.1 we expect there to be a huge amount of mMTC users which are not active all the time. Energy efficiency is desirable for these users, hence, scheduling and grant-based random access is undesirable because of the high overhead cost. Therefore, it is very common to propose grant-free random access schemes for mMTC users [61]. In the massive MIMO case, this will mean that the users send both their pilot and their data in the same message for the base station to decode.

In conventional massive MIMO the users send pilot signals that help the base station to form the channel estimates. The pilots are commonly assumed to be mutually orthogonal, see Section 2.2. In the mMTC case, due to the number of users being huge - larger than the number of samples in the coherence block, $K>\tau_{\mathrm{c}}$, it is not possible to assign orthogonal pilots for all the users.

We can approach this issue in two ways. The first is to use non-orthogonal pilots for the users and hope that the pilot contamination is sufficiently small such that the data is still decodable. Then, the fact that there are much fewer active users than total number of users is used to detect users with compressed sensing $[64,66]$. There is also a possibility of embedding information bits in the pilots, meaning that the data part of the transmission can be eliminated if the information only consists of a few bits [65].

The other option is to use a pilot-hopping pattern, spanning many coherence blocks, to separate users. The pilots are allowed to collide in occasional blocks, but not in all - each user is assigned a unique pilot-hopping sequence, see Figure 5. This scheme relies on the further assumptions that the users are not latency constrained and that there is no DL. This is the scheme that was investigated in Paper B.

\subsubsection{Coverage}

To be able to have a truly smart city, different types of sensors and other mMTC devices need to be able to be placed anywhere. Sometimes they need to be placed in low converge areas, such as basements and remote places. A signal loses much of its power when it needs to go through obstacles, such as walls. The received power affects the receiver's ability to detect and correctly decode the signal.

Low-power wide-area (LPWA) is the terminology used for the networks that deal with these requirements [92]. Because of the inadequacy of cellular 


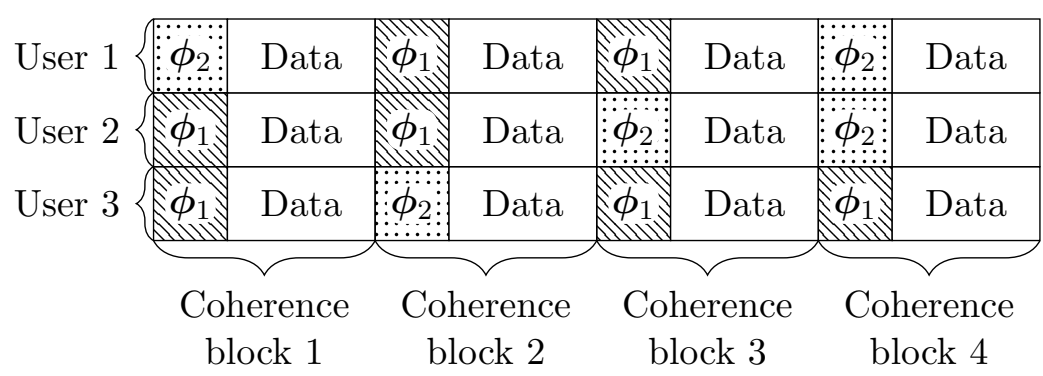

Figure 5: Three users transmitting pilot-hopping sequences of length four using two pilots, $\phi_{1}$ and $\phi_{2}$. Additionally, in each coherence block the users transmit some UL data.

systems that fulfill these requirements, a number of proprietary solutions are available for LPWA networks. Two of the noteworthy proprietary solutions are Sigfox and LoRa which both operate in the unlicensed spectrum [92].

Sigfox is an ultra narrow-band technology and only uses $100 \mathrm{~Hz}$ bandwidth [93]. As a consequence, the data rate is extremely low: only 100 bps. However, using ultra narrow-band brings its benefits: the power consumption and the noise levels are very low. That means that far-away sensors can still communicate and have a long battery life.

LoRa is a spread-spectrum technology which uses a lot more bandwidth than Sigfox [93] but the exact bandwidth changes. LoRa can adaptively change its data rate, which is beneficial if there are devices in different locations. However, Sigfox supports longer ranges than LoRa [94-96].

Massive MIMO will provide a beamforming gain that is proportional to the number of antennas in the base station. This makes the communication link of the remote users stronger and more likely to be stable. By utilizing the beamforming gain, the users can either transmit further with the same power or improve their energy efficiency by lowering the transmit power. Motivated by this, a case study comparing Sigfox, LoRa and a massive MIMO system was made in Paper C. 


\section{Chapter 4}

\section{Future Work}

Although 5G has not yet achieved its full potential, or even been deployed on its own, the research community has started to look beyond $5 \mathrm{G}$ to find the next break through technology for 6G [97-100]. There needs to be researchers working in the far-future technologies but the more present-day, or near-future, problems cannot be forgotten.

In terms of future work pertaining to solving mMTC problems with the massive MIMO technology, there is still work to be done. Below, some extensions based on the included papers are presented.

Deep learning is part of an engineer's toolbox that has become very popular recently. Many applications have been identified in communications, and massive MIMO [101]. It would be interesting to examine if we can beat the algorithms based on approximations in Paper B using deep learning. Further more, it would be very interesting to consider how the algorithms in Paper B translate to cell-free massive MIMO as not all assumptions are the same.

Although, conventional massive MIMO systems assume single-antenna users, the likelihood that this will be the case is not high. Cell phone manufacturers will probably not reduce the number of antennas in the phones in the future. Therefore, it is interesting to investigate signal processing algorithms similar to the ones in Paper A that do not assume that the user has a single antenna. 


\section{Bibliography}

[1] A. Osseiran, F. Boccardi, V. Braun, K. Kusume, P. Marsch, M. Maternia, O. Queseth, M. Schellmann, H. Schotten, H. Taoka, H. Tullberg, M. A. Uusitalo, B. Timus, and M. Fallgren, "Scenarios for 5G mobile and wireless communications: the vision of the METIS project," IEEE Communications Magazine, vol. 52, no. 5, pp. 26-35, May 2014.

[2] IMT Vision - Framework and overall objectives of the future development of IMT for 2020 and beyond, ITU-R Std. M.2083-0, 2015.

[3] H. Chen, R. Abbas, P. Cheng, M. Shirvanimoghaddam, W. Hardjawana, W. Bao, Y. Li, and B. Vucetic, "Ultra-reliable low latency cellular networks: Use cases, challenges and approaches," IEEE Communications Magazine, vol. 56, no. 12, pp. 119-125, Dec. 2018.

[4] K. S. Kim, D. K. Kim, C. Chae, S. Choi, Y. Ko, J. Kim, Y. Lim, M. Yang, S. Kim, B. Lim, K. Lee, and K. L. Ryu, "Ultrareliable and low-latency communication techniques for tactile internet services," Proceedings of the IEEE, vol. 107, no. 2, pp. 376-393, Feb. 2019.

[5] A. Willig, K. Matheus, and A. Wolisz, "Wireless technology in industrial networks," Proceedings of the IEEE, vol. 93, no. 6, pp. 1130-1151, Jun. 2005 .

[6] M. Luvisotto, Z. Pang, and D. Dzung, "Ultra high performance wireless control for critical applications: Challenges and directions," IEEE Transactions on Industrial Informatics, vol. 13, no. 3, pp. 1448-1459, Jun. 2017.

[7] C. Bockelmann, N. Pratas, H. Nikopour, K. Au, T. Svensson, Č. Stefanović, P. Popovski, and A. Dekorsy, "Massive machine-type communications in 5G: physical and MAC-layer solutions," IEEE Communications Magazine, vol. 54, no. 9, pp. 59-65, Sep. 2016.

[8] C. Bockelmann, N. K. Pratas, G. Wunder, S. Saur, M. Navarro, D. Gregoratti, G. Vivier, E. De Carvalho, Y. Ji, Č. Stefanović, P. Popovski, Q. Wang, M. Schellmann, E. Kosmatos, P. Demestichas, M. RacealaMotoc, P. Jung, S. Stanczak, and A. Dekorsy, "Towards massive connectivity support for scalable mMTC communications in 5G networks," IEEE Access, vol. 6, pp. 28 969-28992, 2018. 
[9] F. Boccardi, R. W. Heath, A. Lozano, T. L. Marzetta, and P. Popovski, "Five disruptive technology directions for 5G," IEEE Communications Magazine, vol. 52, no. 2, pp. 74-80, Feb. 2014.

[10] T. L. Marzetta, "Noncooperative cellular wireless with unlimited numbers of base station antennas," IEEE Transactions on Wireless Communications, vol. 9, no. 11, pp. 3590-3600, Nov. 2010.

[11] C. Mollén, "Definition of massive MIMO," 2016. [Online]. Available: http://ma-mimo.ellintech.se/2016/11/04/definition-of-massivemimo/

[12] R. Durrett, Probability: theory and examples. Cambridge University Press, 2010.

[13] S. Malkowsky, J. Vieira, L. Liu, P. Harris, K. Nieman, N. Kundargi, I. C. Wong, F. Tufvesson, V. Öwall, and O. Edfors, "The world's first real-time testbed for massive MIMO: Design, implementation, and validation," IEEE Access, vol. 5, pp. 9073-9088, 2017.

[14] E. Björnson, J. Hoydis, and L. Sanguinetti, "Massive MIMO networks: Spectral, energy, and hardware efficiency," Foundations and Trends® in Signal Processing, vol. 11, no. 3-4, pp. 154-655, 2017.

[15] T. L. Marzetta, E. G. Larsson, H. Yang, and H. Q. Ngo, Fundamentals of Massive MIMO. Cambridge University Press, 2016.

[16] E. Björnson, E. G. Larsson, and M. Debbah, "Massive MIMO for maximal spectral efficiency: How many users and pilots should be allocated?" IEEE Transactions on Wireless Communications, vol. 15, no. 2, pp. 1293-1308, Feb. 2016.

[17] A. Zappone, L. Sanguinetti, G. Bacci, E. Jorswieck, and M. Debbah, "Energy-efficient power control: A look at 5G wireless technologies," IEEE Transactions on Signal Processing, vol. 64, no. 7, pp. 1668-1683, Apr. 2016.

[18] H. V. Cheng, E. Björnson, and E. G. Larsson, "Optimal pilot and payload power control in single-cell massive MIMO systems," IEEE Transactions on Signal Processing, vol. 65, no. 9, pp. 2363-2378, May 2017. 
[19] X. Li, E. Björnson, E. G. Larsson, S. Zhou, and J. Wang, "Massive MIMO with multi-cell MMSE processing: Exploiting all pilots for interference suppression," EURASIP Journal on Wireless Communications and Networking, vol. 2017, no. 1, p. 117, 2017.

[20] E. Nayebi, A. Ashikhmin, T. L. Marzetta, H. Yang, and B. D. Rao, "Precoding and power optimization in cell-free massive MIMO systems," IEEE Transactions on Wireless Communications, vol. 16, no. 7, pp. 4445-4459, Jul. 2017.

[21] H. Yang and T. L. Marzetta, "Massive MIMO with max-min power control in line-of-sight propagation environment," IEEE Transactions on Communications, vol. 65, no. 11, pp. 4685-4693, Nov. 2017.

[22] T. Van Chien, E. Björnson, and E. G. Larsson, "Joint pilot design and uplink power allocation in multi-cell massive MIMO systems," IEEE Transactions on Wireless Communications, vol. 17, no. 3, pp. 2000-2015, Mar. 2018.

[23] A. Ghazanfari, H. V. Cheng, E. Björnson, and E. G. Larsson, "A fair and scalable power control scheme in multi-cell massive MIMO," in ICASSP 2019 - 2019 IEEE International Conference on Acoustics, Speech and Signal Processing (ICASSP), May 2019, pp. 4499-4503.

[24] X. Zhu, Z. Wang, L. Dai, and C. Qian, "Smart pilot assignment for massive MIMO," IEEE Communications Letters, vol. 19, no. 9, pp. 1644-1647, Sep. 2015.

[25] X. Zhu, L. Dai, and Z. Wang, "Graph coloring based pilot allocation to mitigate pilot contamination for multi-cell massive MIMO systems," IEEE Communications Letters, vol. 19, no. 10, pp. 1842-1845, Oct. 2015.

[26] S. Jin, M. Li, Y. Huang, Y. Du, and X. Gao, "Pilot scheduling schemes for multi-cell massive multiple-input-multiple-output transmission," IET Communications, vol. 9, no. 5, pp. 689-700, 2015.

[27] H. Ahmadi, A. Farhang, N. Marchetti, and A. MacKenzie, "A game theoretic approach for pilot contamination avoidance in massive MIMO," IEEE Wireless Communications Letters, vol. 5, no. 1, pp. 12-15, Feb. 2016. 
[28] E. G. Larsson and H. V. Poor, "Joint beamforming and broadcasting in massive MIMO," IEEE Transactions on Wireless Communications, vol. 15, no. 4, pp. 3058-3070, Apr. 2016.

[29] X. Meng, X. Xia, and X. Gao, "Omnidirectional space-time block coding for common information broadcasting in massive MIMO systems," IEEE Transactions on Wireless Communications, vol. 17, no. 3, pp. 1407-1417, Mar. 2018.

[30] M. Karlsson, E. Björnson, and E. G. Larsson, "Performance of in-band transmission of system information in massive MIMO systems," IEEE Transactions on Wireless Communications, vol. 17, no. 3, pp. 1700-1712, Mar. 2018.

[31] Z. Xiang, M. Tao, and X. Wang, "Massive MIMO multicasting in noncooperative cellular networks," IEEE Journal on Selected Areas in Communications, vol. 32, no. 6, pp. 1180-1193, Jun. 2014.

[32] M. Sadeghi, L. Sanguinetti, R. Couillet, and C. Yuen, "Reducing the computational complexity of multicasting in large-scale antenna systems," IEEE Transactions on Wireless Communications, vol. 16, no. 5, pp. 2963-2975, May 2017.

[33] M. Sadeghi, E. Björnson, E. G. Larsson, C. Yuen, and T. L. Marzetta, "Max-min fair transmit precoding for multi-group multicasting in massive MIMO," IEEE Transactions on Wireless Communications, vol. 17, no. 2, pp. 1358-1373, Feb. 2018.

[34] Y. O. Basciftci, C. E. Koksal, and A. Ashikhmin, "Securing massive MIMO at the physical layer," in 2015 IEEE Conference on Communications and Network Security (CNS), Sep. 2015, pp. 272-280.

[35] J. Zhu, R. Schober, and V. K. Bhargava, "Secure transmission in multicell massive MIMO systems," IEEE Transactions on Wireless Communications, vol. 13, no. 9, pp. 4766-4781, Sep. 2014.

[36] D. Kapetanović, G. Zheng, and F. Rusek, "Physical layer security for massive MIMO: An overview on passive eavesdropping and active attacks," IEEE Communications Magazine, vol. 53, no. 6, pp. 21-27, Jun. 2015.

[37] J. Vinogradova, E. Björnson, and E. G. Larsson, "Detection and mitigation of jamming attacks in massive MIMO systems using random 
matrix theory," in 2016 IEEE 17th International Workshop on Signal Processing Advances in Wireless Communications (SPAWC), Jul. 2016, pp. 1-5.

[38] M. Karlsson, E. Björnson, and E. G. Larsson, "Jamming a TDD pointto-point link using reciprocity-based MIMO," IEEE Transactions on Information Forensics and Security, vol. 12, no. 12, pp. 2957-2970, Dec. 2017.

[39] H. Akhlaghpasand, S. M. Razavizadeh, E. Björnson, and T. T. Do, "Jamming detection in massive MIMO systems," IEEE Wireless Communications Letters, vol. 7, no. 2, pp. 242-245, Apr. 2018.

[40] T. T. Do, E. Björnson, E. G. Larsson, and S. M. Razavizadeh, "Jammingresistant receivers for the massive MIMO uplink," IEEE Transactions on Information Forensics and Security, vol. 13, no. 1, pp. 210-223, Jan. 2018.

[41] S. K. Mohammed and E. G. Larsson, "Constant-envelope multi-user precoding for frequency-selective massive mimo systems," IEEE Wireless Communications Letters, vol. 2, no. 5, pp. 547-550, Oct. 2013.

[42] E. Björnson, J. Hoydis, M. Kountouris, and M. Debbah, "Massive MIMO systems with non-ideal hardware: Energy efficiency, estimation, and capacity limits," IEEE Transactions on Information Theory, vol. 60, no. 11, pp. 7112-7139, Nov. 2014.

[43] U. Gustavsson, C. Sanchéz-Perez, T. Eriksson, F. Athley, G. Durisi, P. Landin, K. Hausmair, C. Fager, and L. Svensson, "On the impact of hardware impairments on massive MIMO," in 2014 IEEE Globecom Workshops (GC Wkshps), Dec. 2014, pp. 294-300.

[44] C. Risi, D. Persson, and E. G. Larsson, "Massive MIMO with 1-bit ADC," CoRR, vol. abs/1404.7736, 2014. [Online]. Available: http://arxiv.org/abs/1404.7736

[45] E. Björnson, M. Matthaiou, and M. Debbah, "Massive MIMO with non-ideal arbitrary arrays: Hardware scaling laws and circuit-aware design," IEEE Transactions on Wireless Communications, vol. 14, no. 8, pp. 4353-4368, Aug. 2015.

[46] E. Björnson, M. Matthaiou, A. Pitarokoilis, and E. G. Larsson, "Distributed massive MIMO in cellular networks: Impact of imperfect 
hardware and number of oscillators," in 2015 23rd European Signal Processing Conference (EUSIPCO), Aug. 2015, pp. 2436-2440.

[47] Y. Zou, O. Raeesi, L. Antilla, A. Hakkarainen, J. Vieira, F. Tufvesson, Q. Cui, and M. Valkama, "Impact of power amplifier nonlinearities in multi-user massive MIMO downlink," in 2015 IEEE Globecom Workshops (GC Wkshps), Dec. 2015, pp. 1-7.

[48] C. Mollén, E. G. Larsson, and T. Eriksson, "Waveforms for the massive MIMO downlink: Amplifier efficiency, distortion, and performance," IEEE Transactions on Communications, vol. 64, no. 12, pp. 5050-5063, Dec. 2016.

[49] D. Verenzuela, E. Björnson, and M. Matthaiou, "Hardware design and optimal ADC resolution for uplink massive MIMO systems," in 2016 IEEE Sensor Array and Multichannel Signal Processing Workshop (SAM), Jul. 2016, pp. 1-5.

[50] S. Jacobsson, G. Durisi, M. Coldrey, U. Gustavsson, and C. Studer, "Throughput analysis of massive MIMO uplink with low-resolution ADCs," IEEE Transactions on Wireless Communications, vol. 16, no. 6, pp. 4038-4051, Jun. 2017.

[51] M. Sarajlić, L. Liu, and O. Edfors, "When are low resolution ADCs energy efficient in massive MIMO?" IEEE Access, vol. 5, pp. 14837$14853,2017$.

[52] S. Buzzi and C. D'Andrea, "Cell-free massive MIMO: User-centric approach," IEEE Wireless Communications Letters, vol. 6, no. 6, pp. 706-709, Dec. 2017.

[53] H. Q. Ngo, A. Ashikhmin, H. Yang, E. G. Larsson, and T. L. Marzetta, "Cell-free massive MIMO versus small cells," IEEE Transactions on Wireless Communications, vol. 16, no. 3, pp. 1834-1850, Mar. 2017.

[54] M. Bashar, K. Cumanan, A. G. Burr, H. Q. Ngo, and M. Debbah, "Cellfree massive MIMO with limited backhaul," in 2018 IEEE International Conference on Communications (ICC), May 2018, pp. 1-7.

[55] Z. Chen and E. Björnson, "Channel hardening and favorable propagation in cell-free massive MIMO with stochastic geometry," IEEE Transactions on Communications, vol. 66, no. 11, pp. 5205-5219, Nov. 2018. 
[56] G. Interdonato, E. Björnson, H. Q. Ngo, P. Frenger, and E. G. Larsson, "Ubiquitous cell-free massive MIMO communications," EURASIP Journal on Wireless Communications and Networking, vol. 2019, no. 1, p. 197, 2019.

[57] P. Popovski, J. J. Nielsen, Č. Stefanović, E. d. Carvalho, E. Ström, K. F. Trillingsgaard, A. Bana, D. M. Kim, R. Kotaba, J. Park, and R. B. Sørensen, "Wireless access for ultra-reliable low-latency communication: Principles and building blocks," IEEE Network, vol. 32, no. 2, pp. 16-23, Mar. 2018.

[58] A.-S. Bana, E. de Carvalho, B. Soret, T. Abrão, J. C. Marinello, E. G. Larsson, and P. Popovski, "Massive MIMO for internet of things (IoT) connectivity," Physical Communication, vol. 37, p. 100859, 2019. [Online]. Available: http://www.sciencedirect.com/science/article/pii/S1874490719303891

[59] A. Kosta, N. Pappas, and V. Angelakis, "Age of information: A new concept, metric, and tool," Foundations and Trends® in Networking, vol. 12 , no. 3, pp. 162-259, 2017. [Online]. Available: http://dx.doi.org/10.1561/1300000060

[60] E. d. Carvalho, E. Björnson, J. H. Sørensen, P. Popovski, and E. G. Larsson, "Random access protocols for massive MIMO," IEEE Communications Magazine, vol. 55, no. 5, pp. 216-222, May 2017.

[61] L. Liu, E. G. Larsson, W. Yu, P. Popovski, Č. Stefanović, and E. de Carvalho, "Sparse signal processing for grant-free massive connectivity: A future paradigm for random access protocols in the internet of things," IEEE Signal Processing Magazine, vol. 35, no. 5, pp. 88-99, Sep. 2018.

[62] H. Q. Ngo, E. G. Larsson, and T. L. Marzetta, "Energy and spectral efficiency of very large multiuser MIMO systems," IEEE Transactions on Communications, vol. 61, no. 4, pp. 1436-1449, Apr. 2013.

[63] E. Björnson, E. de Carvalho, J. H. Sørensen, E. G. Larsson, and P. Popovski, "A random access protocol for pilot allocation in crowded massive MIMO systems," IEEE Transactions on Wireless Communications, vol. 16, no. 4, pp. 2220-2234, Apr. 2017.

[64] L. Liu and W. Yu, "Massive connectivity with massive MIMO - part I: Device activity detection and channel estimation," IEEE Transactions on Signal Processing, vol. 66, no. 11, pp. 2933-2946, Jun. 2018. 
[65] K. Senel and E. G. Larsson, "Grant-free massive MTC-enabled massive MIMO: A compressive sensing approach," IEEE Transactions on Communications, vol. 66, no. 12, pp. 6164-6175, Dec. 2018.

[66] S. Haghighatshoar, P. Jung, and G. Caire, "Improved scaling law for activity detection in massive MIMO systems," CoRR, vol. abs/1803.02288, 2018. [Online]. Available: http://arxiv.org/abs/1803.02288

[67] P. Popovski, K. F. Trillingsgaard, O. Simeone, and G. Durisi, "5G wireless network slicing for eMBB, URLLC, and mMTC: A communicationtheoretic view," IEEE Access, vol. 6, pp. 55 765-55 779, 2018.

[68] K. Senel, E. Björnson, and E. G. Larsson, "Humans and machines can be jointly spatially multiplexed by massive MIMO," CoRR, vol. abs/1808.09177, 2018. [Online]. Available: http://arxiv.org/abs/1808.09177

[69] T. S. Rappaport, Wireless communications: principles and practice., ser. Prentice Hall communications engineering and emerging technologies series. Prentice Hall PTR, 2002.

[70] R. W. Heath Jr and A. Lozano, Foundations of MIMO communication. Cambridge University Press, 2018.

[71] D. Tse and P. Viswanath, Fundamentals of wireless communication. Cambridge University Press, 2005.

[72] U. Madhow, Fundamentals of Digital Communication. Cambridge University Press, 2008.

[73] C. A. Balanis, Antenna Theory: Analysis and Design. Wiley, 2016, vol. Fourth edition.

[74] J. Vieira, F. Rusek, O. Edfors, S. Malkowsky, L. Liu, and F. Tufvesson, "Reciprocity calibration for massive MIMO: Proposal, modeling, and validation," IEEE Transactions on Wireless Communications, vol. 16, no. 5, pp. 3042-3056, May 2017.

[75] Chang-Fa Yang, Boau-Cheng Wu, and Chuen-Jyi Ko, "A ray-tracing method for modeling indoor wave propagation and penetration," IEEE Transactions on Antennas and Propagation, vol. 46, no. 6, pp. 907-919, Jun. 1998. 
[76] J. H. Tarng, Wen-Shun Liu, Yeh-Fong Huang, and Jiunn-Ming Huang, "A novel and efficient hybrid model of radio multipath-fading channels in indoor environments," IEEE Transactions on Antennas and Propagation, vol. 51, no. 3, pp. 585-594, Mar. 2003.

[77] O. Ledoit and M. Wolf, "A well-conditioned estimator for large-dimensional covariance matrices," Journal of Multivariate Analysis, vol. 88, no. 2, pp. 365 - 411, 2004. [Online]. Available: http://www.sciencedirect.com/science/article/pii/S0047259X03000964

[78] E. Björnson, L. Sanguinetti, and M. Debbah, "Massive MIMO with imperfect channel covariance information," in 2016 50th Asilomar Conference on Signals, Systems and Computers, Nov. 2016, pp. 974978.

[79] K. Upadhya and S. A. Vorobyov, "Covariance matrix estimation for massive MIMO," IEEE Signal Processing Letters, vol. 25, no. 4, pp. 546-550, Apr. 2018.

[80] D. Neumann, M. Joham, and W. Utschick, "Covariance matrix estimation in massive MIMO," IEEE Signal Processing Letters, vol. 25, no. 6, pp. 863-867, Jun. 2018.

[81] Ö. Özdogan, E. Björnson, and E. G. Larsson, "Massive MIMO with spatially correlated Rician fading channels," IEEE Transactions on Communications, vol. 67, no. 5, pp. 3234-3250, May 2019.

[82] K. Upadhya, S. A. Vorobyov, and M. Vehkapera, "Superimposed pilots are superior for mitigating pilot contamination in massive MIMO," IEEE Transactions on Signal Processing, vol. 65, no. 11, pp. 2917-2932, Jun. 2017.

[83] D. Verenzuela, E. Björnson, and L. Sanguinetti, "Spectral and energy efficiency of superimposed pilots in uplink massive MIMO," IEEE Transactions on Wireless Communications, vol. 17, no. 11, pp. 70997115, Nov. 2018.

[84] O. Elijah, C. Y. Leow, T. A. Rahman, S. Nunoo, and S. Z. Iliya, "A comprehensive survey of pilot contamination in massive MIMO $-5 \mathrm{G}$ system," IEEE Communications Surveys Tutorials, vol. 18, no. 2, pp. 905-923, Second Quarter 2016.

[85] S. M. Kay, Fundamentals of statistical signal processing, ser. PrenticeHall signal processing series. Prentice Hall PTR, 1993. 
[86] "Ericsson Mobility Report June 2019," Ericsson, 2019.

[87] C. Perera, A. Zaslavsky, P. Christen, and D. Georgakopoulos, "Context aware computing for the internet of things: A survey," IEEE Communications Surveys Tutorials, vol. 16, no. 1, pp. 414-454, First Quarter 2014.

[88] S. Andreev, O. Galinina, A. Pyattaev, M. Gerasimenko, T. Tirronen, J. Torsner, J. Sachs, M. Dohler, and Y. Koucheryavy, "Understanding the IoT connectivity landscape: a contemporary M2M radio technology roadmap," IEEE Communications Magazine, vol. 53, no. 9, pp. 32-40, Sep. 2015.

[89] A. Zanella, N. Bui, A. Castellani, L. Vangelista, and M. Zorzi, "Internet of things for smart cities," IEEE Internet of Things Journal, vol. 1, no. 1, pp. 22-32, Feb. 2014.

[90] L. D. Xu, W. He, and S. Li, "Internet of things in industries: A survey," IEEE Transactions on Industrial Informatics, vol. 10, no. 4, pp. 2233-2243, Nov. 2014.

[91] E. Dahlman, S. Parkvall, and J. Sköld, 5G NR: The next generation wireless access technology. Academic Press, 2018.

[92] U. Raza, P. Kulkarni, and M. Sooriyabandara, "Low power wide area networks: An overview," IEEE Communications Surveys Tutorials, vol. 19, no. 2, pp. 855-873, Second Quarter 2017.

[93] W. Yang, M. Wang, J. Zhang, J. Zou, M. Hua, T. Xia, and X. You, "Narrowband wireless access for low-power massive internet of things: A bandwidth perspective," IEEE Wireless Communications, vol. 24, no. 3 , pp. 138-145, Jun. 2017.

[94] J. Petajajarvi, K. Mikhaylov, A. Roivainen, T. Hanninen, and M. Pettissalo, "On the coverage of LPWANs: range evaluation and channel attenuation model for lora technology," in 2015 14th International Conference on ITS Telecommunications (ITST), Dec. 2015, pp. 55-59.

[95] K. E. Nolan, W. Guibene, and M. Y. Kelly, "An evaluation of low power wide area network technologies for the internet of things," in 2016 International Wireless Communications and Mobile Computing Conference (IWCMC), Sep. 2016, pp. 439-444. 
[96] B. Vejlgaard, M. Lauridsen, H. Nguyen, I. Z. Kovacs, P. Mogensen, and M. Sørensen, "Coverage and capacity analysis of Sigfox, LoRa, GPRS, and NB-IoT," in 2017 IEEE 85th Vehicular Technology Conference (VTC Spring), Jun. 2017, pp. 1-5.

[97] K. David and H. Berndt, "6G vision and requirements: Is there any need for beyond 5G?" IEEE Vehicular Technology Magazine, vol. 13, no. 3, pp. 72-80, Sep. 2018.

[98] W. Saad, M. Bennis, and M. Chen, "A vision of $6 \mathrm{G}$ wireless systems: Applications, trends, technologies, and open research problems," IEEE Network, pp. 1-9, 2019.

[99] T. S. Rappaport, Y. Xing, O. Kanhere, S. Ju, A. Madanayake, S. Mandal, A. Alkhateeb, and G. C. Trichopoulos, "Wireless communications and applications above 100 ghz: Opportunities and challenges for 6G and beyond," IEEE Access, vol. 7, pp. 78 729-78 757, 2019.

[100] E. Björnson, L. Sanguinetti, H. Wymeersch, J. Hoydis, and T. L. Marzetta, "Massive MIMO is a reality-what is next?: Five promising research directions for antenna arrays," Digital Signal Processing, vol. 94, pp. 3 - 20, 2019, special Issue on Source Localization in Massive MIMO. [Online]. Available: http://www.sciencedirect.com/science/article/pii/S1051200419300776

[101] M. Chen, U. Challita, W. Saad, C. Yin, and M. Debbah, "Artificial neural networks-based machine learning for wireless networks: A tutorial," IEEE Communications Surveys Tutorials, vol. 21, no. 4, pp. 3039-3071, Fourth Quarter 2019. 


\section{Included Papers}





\section{Papers}

The papers associated with this thesis have been removed for copyright reasons. For more details about these see:

http://urn.kb.se/resolve?urn=urn:nbn:se:liu:diva-162586 


\section{Other Recently Published Theses From \\ The Division of Communication Systems Department of Electrical Engineering (ISY) Linköping University, Sweden}

Daniel Verenzuela, Exploring Alternative Massive MIMO Designs: Superimposed Pilots and Mixed-ADCs, Linköping Studies in Science and Technology. Dissertations, No. 2041, 2020.

Trịnh Văn Chiến, Spatial Resource Allocation in Massive MIMO Communication: From Cellular to Cell-Free, Linköping Studies in Science and Technology. Dissertations, No. 2036, 2020.

Amin Ghazanfari, Power Control for Multi-Cell Massive MIMO, Linköping Studies in Science and Technology. Licentiate Thesis, No. 1852, 2019.

Giovanni Interdonato, Signal Processing Aspects of Cell-Free Massive MIMO, Linköping Studies in Science and Technology. Licentiate Thesis, No. 1817, 2018.

Marcus Karlsson, Blind Massive MIMO Base Stations: Downlink Transmission and Jamming, Linköping Studies in Science and Technology. Dissertations, No. 1950, 2018.

Victor Hei Cheng, Optimizing Massive MIMO: Precoder Design and Power Allocation, Linköping Studies in Science and Technology. Dissertations, No. 1929, 2018.

Christopher Mollén, High-End Performance with Low-End Hardware: Analysis of Massive MIMO Base Station Transceivers, Linköping Studies in Science and Technology. Dissertations, No. 1896, 2017.

Antonios Pitarokoilis, Phase Noise and Wideband Transmission in Massive MIMO, Linköping Studies in Science and Technology. Dissertations, No. 1756, 2016.

Anu Kalidas M. Pillai, Signal Reconstruction Algorithms for Time-Interleaved ADCs, Linköping Studies in Science and Technology. Dissertations, No. 1672, 2015.

Ngô Quốc Hiển, Massive MIMO: Fundamentals and System Designs, Linköping Studies in Science and Technology. Dissertations, No. 1642, 2015.

Mirsad Čirkić, Efficient MIMO Detection Methods, Linköping Studies in Science and Technology. Dissertations, No. 1570, 2014.

Reza Moosavi, Improving the Efficiency of Control Signaling in Wireless Multiple Access Systems, Linköping Studies in Science and Technology. Dissertations, No. 1556, 2014. 


\section{FACULTY OF SCIENCE AND ENGINEERING}

Linköping Studies in Science and Technology,

Licentiate Thesis No. 1868, 2020

Division of Communication Systems

Department of Electrical Engineering

Linköping University

SE-581 83 Linköping, Sweden

www.liu.se 